\title{
A cyclin-dependent kinase inhibitor, dinaciclib, impairs homologous recombination and sensitizes multiple myeloma cells to PARP inhibition
}

\author{
David A. Alagpulinsa ${ }^{1,2}$, Srinivas Ayyadevara ${ }^{1,2}$, Shmuel Yaccoby $^{3}$, and Robert J. \\ Shmookler Reis ${ }^{1,2, *}$ \\ ${ }^{1}$ McClellan Veterans Medical Center, Central Arkansas Veterans Healthcare System, Little Rock \\ AR 72205, USA \\ ${ }^{2}$ Dept. of Geriatrics, University of Arkansas for Medical Science, Little Rock, AR 72205, USA \\ ${ }^{3}$ Myeloma Institute for Research and Therapy, University of Arkansas for Medical Sciences, Little \\ Rock, AR 72205, USA
}

\begin{abstract}
Poly(ADP-ribose) polymerases 1 and 2 (PARP1/2) are required for single-strand break repair, and their inhibition causes DNA replication-fork collapse and double-strand break (DSB) formation. These DSBs are primarily repaired via homologous recombination (HR), a high-fidelity repair pathway. Should HR be deficient, DSBs may be repaired via error-prone nonhomologous end joining mechanisms, or may persist, ultimately resulting in cell death. Synthetic lethality thus exists between PARP and HR functions. Multiple myeloma (MM) cells are characterized by chromosomal instability and pervasive DNA damage, implicating aberrant DNA repair. Cyclindependent kinases (CDKs), upstream modulators of HR, are dysregulated in MM. Here we show that a CDK inhibitor, dinaciclib, impairs HR repair and sensitizes MM cells to the PARP1/2 inhibitor ABT-888. Dinaciclib abolishes ABT-888-induced BRCA1 and RAD51 foci and potentiates DNA damage, indicated by increased $\gamma \mathrm{H} 2 \mathrm{AX}$ foci. Dinaciclib treatment reduces expression of HR-repair genes, including Rad51, and blocks BRCA1 phosphorylation, a modification required for HR repair, thus inhibiting HR repair of chromosome DSBs. Cotreatment with dinaciclib and ABT-888 in vitro resulted in synthetic lethality of MM cells, but not normal CD19+ B cells, and slowed growth of MM xenografts in SCID mice almost two-fold. These findings support combining dinaciclib with PARP inhibitors for MM therapy.
\end{abstract}

\section{Keywords}

Multiple myeloma; DNA damage; synthetic lethality; cyclin-dependent kinase; poly(ADP-ribose) polymerase; homologous recombination

*Correspondence: Robert J. Shmookler Reis, 629 Jack Stephens Drive, Little Rock, AR 72205, USA, rjsr@uams.edu, Phone: +1 501-960-3031; Fax: +1 501-526-5830.

Conflict of Interest: The authors declare no conflict of interest. 


\section{Introduction}

Multiple myeloma (MM) is a hematologic malignancy characterized by clonal plasma-cell proliferation in the bone marrow (1), and widespread chromosomal instability in those cells (2). Although the mechanisms underlying genomic instability in MM are not clearly defined, deranged DNA repair mechanisms have been implicated (3-5). Cells employ several DNA repair mechanisms to withstand genotoxic stress. The most lethal DNA lesions, doublestrand breaks (DSBs), are repaired by one of two major pathways-nonhomologous endjoining (NHEJ) and homologous recombination (HR). The mechanism of NHEJ is simpler (direct ligation of DSB ends), but the process is more error-prone in the absence of sequence homology to guide repair (6). HR repair instead uses a sister chromatid or more rarely, a non-sister homolog, as template for high-fidelity repair, and thus occurs mainly in the $\mathrm{S}$ and G2 cell cycle phases when a sister chromatid is available (7). During HR repair, DSBs can be detected by the MRE11-RAD50-NBS1 (MRN) complex, which recruits and activates ATM (8). Activated ATM phosphorylates multiple proteins involved in initiating repair and checkpoint arrest, including H2AX, MDCI, CHK2 and BRCA1 (8-10). Cyclin-dependent kinase (CDK)-mediated phosphorylation activates CtIP, enabling it to interact with BRCA1 to promote resection of DSB termini, thus generating $3^{\prime}$-single strand DNA (ssDNA) tails $(11,12)$. With the aid of BRCA2, RAD51 binds to ssDNA to form RAD51 nucleofilaments, which orchestrate a homology search on a template (e.g. a sister chromatid), strand invasion, and repair of the DSB (13).

DNA single-strand breaks (SSBs) are repaired via the base or nucleotide excision-repair pathway and require poly(ADP-ribose) polymerase 1 (PARP1), and PARP2 to some extent (14). Inhibition of PARP1/2 therefore leads to accumulation of SSBs that cause collapse of DNA replication forks and formation of lethal DSBs (15). These replication-associated DSBs are primarily repaired via HR, the high-fidelity repair pathway for DSBs (16). In the absence of HR the DSBs persist, unless repaired by error-prone NHEJ mechanisms, ultimately resulting in cell death $(14,17,18)$. Synthetic lethality thus exists between PARP1 and HR function, which has been exploited to selectively kill cells that harbor HR deficiencies due to mutations in their BRCA genes $(15,18)$. Expression of PARP1 and the frequency of HR are both elevated in MM cells $(3,19)$, whereas defective DNA-repair mechanisms, including NHEJ $(4,20)$ and mismatch repair (MMR) (5), have been reported. Unlike normal bone marrow plasma cells and normal B cells, MM cell lines and patient plasma cells exhibit pervasive DNA damage and rearrangements (21). Thus MM cells may be highly dependent on PARP1 and HR functions to survive oncogene-induced replication stress and the DNA damage that ensues. Consequently, concomitant inhibition of PARP1 and HR results in a contextual synthetic lethality of MM cells, but not of normal CD34 ${ }^{+}$ hematopoietic stem cells (19).

Recent studies have revealed critical roles for CDKs in regulating HR; however, CDK dysregulation is a hallmark of MM cells and CDKs have been designated as therapeutic targets (22-24). CDK1 and CDK2 phosphorylate BRCA1 on serine 1497 (S1497) $(25,26)$ and in addition, CDK1 phosphorylates BRCA1 on S1189/1191 (26). These posttranslational modifications are important for formation of BRCA1 and RAD51 foci in response to DNA damage and $\mathrm{HR}$ repair $(26,27)$. Also, CDK5 and its activator, CDK5R/ 
p35, are overexpressed in MM cells relative to normal somatic tissue $(28,29)$. This CDK can phosphorylate and activate ATM, which is critical for activation of the intra-S and G2/M checkpoints following DNA damage $(28,30)$. Consequently, cancer cells depleted of either CDK1 or CDK5 become hypersensitive to PARP inhibitors $(28,31)$ whereas upregulation of CDK5 can mediate chemoresistance (32). Due to the critical roles CDKs play in DNA repair by HR, CDK inhibitors such as flavopiridol, AG024322 and AZD5438 have been shown to impair HR function and sensitize cancer cells to DNA damaging agents, including PARP inhibitors (33-35).

Dinaciclib is a potent small-molecule inhibitor of CDKs 1, 2, 5 and 9 (36). It has shown promising results in early-phase clinical trials for MM (24). In this study, we postulated that dinaciclib could disrupt HR repair in MM cells, leading to a contextual synthetic lethality if combined with PARP1/2 inhibition. We here provide evidence that dinaciclib does impair HR repair efficiency in MM cells and sensitizes them to PARP inhibition both in vitro and in vivo.

\section{Materials and Methods}

\section{Cell culture and treatments}

The human MM cell lines NCI-H929, RPMI 8226 and MM.1S were purchased from ATCC and authenticated by them; all were used within $<6$ months of post-receipt cultivation. Cells were maintained in RPMI 1640 medium with L-glutamine and $\mathrm{NaHCO}_{3}$ (ATCC) containing $10 \%$ FBS (ATCC), 100U/mL of penicillin and $100 \mu \mathrm{g} / \mathrm{mL}$ of streptomycin (Sigma-Aldrich). MM.1S-DR.GFP cells (kindly provided by Dr. Nizar Bahlis, Univ. of Calgary, Canada), were derived from the MM.1S line but not authenticated by us, were maintained in the same medium but supplemented with $2 \mu \mathrm{g} / \mathrm{mL}$ puromycin (Sigma-Aldrich). Normal human peripheral blood CD19+ $\mathrm{B}$ cells (ZenBio) were cultured in ZenBio Lymphocyte medium.

ABT-888 (Santa Cruz Biotechnology, Inc.), doxorubicin-HCl (Sigma Aldrich) and dinaciclib (SCH727965; ChemieTek) were dissolved in DMSO. The stock drug concentrations were diluted in cell-culture medium prior to cell treatment. For in vivo studies, dinaciclib was dissolved in $15 \%$ Captisol $^{\circledR}$ (Ligand Pharmaceuticals, Inc.).

\section{Flow-cytometry analysis of cell-cycle distributions, bromodeoxyuridine (BrdU) incorporation, and histone H3 (S10) phosphorylation}

After the indicated treatments, cell-cycle distribution was analyzed, and phosphorylated histone H3 (S10) was visualized by immunostaining as previously described (37).

For propidium iodide (PI) and BrdU double staining, cells were incubated with $10 \mu \mathrm{M}$ BrdU (Sigma) at $37^{\circ} \mathrm{C}$ for $1 \mathrm{~h}$, fixed in $70 \%$ ethanol, denatured in $2-\mathrm{M} \mathrm{HCl}$, and neutralized in 0.1 $\mathrm{M}$ sodium borate. Cells were then stained with FITC-labeled antibody to BrdU (BD Pharmigen, San Diego, CA), resuspended in $500 \mu \mathrm{L}$ PBS containing $25 \mu \mathrm{g} / \mathrm{mL}$ PI and 1.25 $\mathrm{mg} / \mathrm{mL}$ RNase $\mathrm{A}$, and incubated at $37^{\circ} \mathrm{C}$ for $30 \mathrm{~min}$ in the dark. Nuclear staining was then quantified by flow cytometry (FACScan), using FlowJo 4.4.4 software for DNA-content analysis. 


\section{Real-Time PCR (RT-qPCR)}

Total RNA was extracted from cells after the indicated treatments using RNAeasy Mini Kit (Qiagen). Total RNA (1 $\mu \mathrm{g})$ was used to reverse transcribe cDNA using SuperScript First Strand cDNA synthesis kit (Invitrogen). The cDNA was amplified by RT-qPCR using an ABI Prism 7900 HT Sequence Detection system (Applied Biosystems). The amplified PCR products were detected using SYBR Green Master Mix (Roche). PCRs of the Gapdh gene served as internal controls; thus the threshold cycle number $\left(T_{c}\right)$ for each sample was normalized to $\mathrm{T}_{\mathrm{c}}$ for GAPDH. The mRNA levels in treated samples were standardized against samples exposed to DMSO control. The forward (F) and reverse (R) primers used for amplification were:

Rad51: 5'- CAAtGCAGAtGCAGCTtGAA-3' (F) and 5'- CCtTGGCtTCACTAAttCCCT-3' (R).

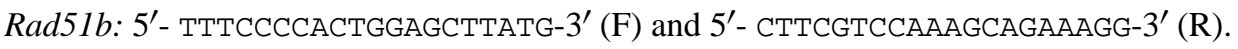

Rad51c: 5'- AgACGtTCCGCtttgaAatg-3' (F) and 5'- GgAgttcCtCAGCAgtCtGG-3' (R).

Rad51d: 5'- AgTGGTGGACCTGGTtTCTG-3' (F) and 5'- CCAAGTCCTGCCTTCtTCAG-3' (R).

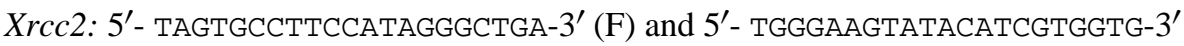

(R).

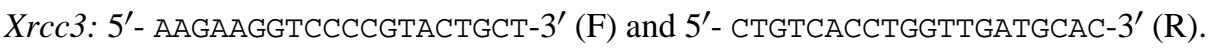

Brcal: 5' - TAGGGCTGGAAGCACAGAGT-3' (F), 5'- AAtTtCCTCCCCAAtGTtCC-3' (R)

Gapdh: 5'- GTCCACTGGCGTCTTCACCA-3' (F), 5'- GTGGCAGTGATGGCATGGAC-3' (R)

\section{Western blots}

After the indicated treatments, whole cell lysates were prepared in cell lysis buffer (Cell Signaling Technology) supplemented with protease and phosphatase inhibitor cocktails (Thermo Scientific). The supernatants were collected after centrifugation at 14,000 rpm at $4^{\circ} \mathrm{C}$ for $10 \mathrm{~min}$. Protein concentrations were determined using the BCA assay kit (Thermo Scientific). Equal protein loads, from all samples in an experiment, were heated for $5 \mathrm{~min}$ at $95^{\circ} \mathrm{C}$ in Laemmli buffer (Bio-Rad), resolved by SDS-PAGE, transferred to a polyvinyl difluoride membrane (PVDF, Millipore), and probed with antibodies recognizing RAD51 (mouse monoclonal IgG, Millipore), and p-BRCA1 (S1497, rabbit polyclonal IgG, Santa Cruz Biotech.). Membranes were washed in TBST, incubated for $1 \mathrm{~h}$ at $22^{\circ} \mathrm{C}$ with HRPconjugated anti-mouse IgG and anti-rabbit IgG (Santa Cruz Biotechnology), and washed again. Protein signals were detected by chemiluminescence using an ECL detection reagent (Bio-Rad). After removing antibodies in stripping buffer $(2 \% \mathrm{w} / \mathrm{v}$ sodium dodecyl sulfate [SDS]; 62.5-mM Tris-HCl pH 6.8; and 0.7\% [w/w] $\beta$-mercaptoethanol) for $15 \mathrm{~min}$ at $50^{\circ} \mathrm{C}$, the membrane was re-probed with primary antibodies to BRCA1 and $\beta$-actin (Santa Cruz Biotechnology) and detected as above. RAD51 and pBRCA1 protein band densities were quantified using Quantity One software (Bio-Rad) and the values expressed relative to actin and total BRCA1, respectively (controls for loading and transfer). The protein levels in treated samples were standardized against controls exposed only to DMSO. At least two independent experiments were performed for each comparison. 


\section{Immunofluorescence cell staining and confocal microscopy}

Immunofluorescence cell staining and confocal microscopy were carried out as previously described (37). Cells were incubated with primary antibodies against $\gamma \mathrm{H} 2 \mathrm{AX}$ (ser139; 1:1000 mouse monoclonal IgG, clone JBW301, Millipore) and either RAD51 (goat polyclonal IgG; Santa Cruz Biotech.) or rabbit polyclonal anti-BRCA1 IgG (1:1000 rabbit polyclonal IgG, Santa Cruz Biotech.) at $4^{\circ} \mathrm{C}$ overnight. After three 5-min washes with PBS, cells were incubated $1 \mathrm{~h}$ at $22^{\circ} \mathrm{C}$ in the dark, with appropriate secondary antibodies at 1:1000 dilutions (bovine anti-goat IgG Alexa Fluor 488 for RAD51, or donkey anti-rabbit IgG FITC and goat anti-mouse IgG Alexa Fluor 594 for $\gamma \mathrm{H} 2 \mathrm{AX}$ [Jackson ImmunoResearch]). Cells were washed 3 times in PBS and mounted under coverslips with Prolong Antifade reagent containing DAPI. Images were acquired with an LSM 510 Zeiss confocal laser-scanning microscope with a $63 \times$ oil objective. For quantitative analysis, $\geq 100$ cells from each group were chosen at random and nuclei were (a.) counted manually to determine the percent positive for BRCA1 or RAD51 or $\gamma \mathrm{H} 2 \mathrm{AX}$, based on a threshold of 25 discrete foci per nucleus; and (b.) quantified from fluorescence images to obtain the total integrated pixel intensity, from which the background was subtracted (perimeter signal per pixel multiplied by total area pixels). Results were averaged from at least 3 biological replicates.

\section{Homologous recombination repair assay}

The effect of dinaciclib on HR repair was assessed as previously described (37).

\section{Cell proliferation and colony formation assays}

Cell proliferation/viability and colony formation assays were performed as previously described (37). The percent viability of cells was calculated relative to vehicle treatment (set as $100 \%$ viability for dose-response curves).

\section{Myeloma xenograft mouse model}

The animal study was approved by the Animal Use and Care Committee of the University of Arkansas for Medical Sciences. The xenograft model used here has been described previously (38). Briefly, CB-17/SCID male mice (Harlan Laboratories Ltd.) were subcutaneously inoculated with $5.0 \times 10^{6} \mathrm{MM}$.1S cells in 50\% matrigel (Corning) in serumfree RPMI 1640 cell culture medium. Approximately two weeks after cell inoculation, when tumors became measurable $\left(100-150 \mathrm{~mm}^{3}\right)$, mice were assessed for initial tumor size, randomized into four groups (each $\mathrm{N}=10$ ) and treated as follows:

i. $\operatorname{DMSO}(6 \%, \mathrm{v} / \mathrm{v}$, intraperitoneally (i.p) twice per week)

ii. Dinaciclib (35 mg/kg i.p twice per week) (36)

iii. ABT-888 (50 mg/kg by oral gavage, twice daily, 5 days per week) (19)

iv. The combination of ii and iii.

The long-axis $(a)$ and short-axis $(b)$ diameters of tumors were measured by caliper every third day to estimate tumor volume using the formula: $V=0.5 a \times b^{2}$ (ref. (19)). For survival analysis, survival time was defined as time required for tumor volume to reach a set 
endpoint, i.e. tumor growth exceeding $2 \mathrm{~cm}$ across the long-axis, or causing major distress to the mice. Differences in tumor volume among treatment groups were tested for significance by one-way ANOVA with Tukey's post hoc tests. Survival was determined from KaplanMeier curves, as the time from the first day of treatment until mice were sacrificed (based on the above criteria). Statistical significance of survival differences was determined by a logrank (Mantel-Cox) test.

\section{Statistical analysis}

GraphPad Prism software (Prism ver. 6, San Diego, CA, USA) and Excel were used for statistical analysis. Statistical significances of differences between groups were calculated by the Fisher $t$ test, requiring $p<0.05$ for nominal significance.

\section{Results}

\section{Co-treatment with the CDK inhibitor dinaciclib and the PARP1/2 inhibitor ABT-888 causes synthetic lethality of human myeloma cell lines, but not of normal peripheral $B$ cells}

Treatment of MM cells with the PARP1/2 inhibitor ABT- 888 has been shown to induce DNA DSBs that are effectively repaired via HR, and therefore do not affect cell viability (19). Because we hypothesized that the CDK inhibitor dinaciclib would impair HR repair and hence sensitize MM cells to PARP inhibition, we first assessed cell viability under growth conditions, and clonogenic survival at low density, of MM cells treated with ABT-888 or dinaciclib as single agents vs. their combination. As shown in Figure 1, A-C and E-F, treatment with ABT-888 (2.5-20 $\mu \mathrm{M})$ alone had no significant effect (less than $10 \%$; each $p>0.05$ ) on cell viability in either assay, for any of the MM cell lines tested. Dinaciclib was also tested across a dose range, 5-40 nM, but data will be shown here only for $20 \mathrm{nM}$ (a dose slightly above the $\mathrm{IC}_{50}$, which produced maximum sensitization to the PARP inhibitor). Dinaciclib caused significant inhibitions of both viability and colony formation, which were similar (47-55\% reduction; each $p \unlhd 0.01$ ) for all of the MM cell lines tested. The combination of dinaciclib with ABT-888, however, produced synergistic effects on cell survival (synthetic lethality), similarly dependent on ABT-888 dose for every MM cell line (Figure 1, A-C). A statistical criterion for synergy, that the viable fraction for dual treatment must be significantly lower (at $p<0.05$ ) than the product of viable fractions after individual treatments, was met for 9 of the 12 non-zero ABT- 888 treatments (Figure 1, AC) in mass-culture conditions, and for both combined treatments under clonal-growth conditions (Figure 1, E-F). We observed similarly pronounced synergistic effects when dinaciclib was combined with low doses of doxorubicin (20-160 nM), which induces DSBs (Supplementary Figure 1, A-B). Moreover, a small-molecule inhibitor of RAD51, B02, which blocks HR repair in MM cells (37), also sensitized MM.1S cells to ABT-888 (Figure $1 \mathrm{G})$, demonstrating that HR suppression (rather than a direct drug interaction) is responsible for synthetic lethality. In contrast, untransformed $\mathrm{CD} 19^{+} \mathrm{B}$ cells, isolated from normal human peripheral blood, evinced no dose-dependent rise in cell killing by ABT-888 (at 2.5$20 \mu \mathrm{M}$ ) in the presence of $20-\mathrm{nM}$ dinaciclib), with the viable fraction staying very close to 75\%; there was thus no indication of synergy between these drugs in normal B cells (Figure 1D). 


\section{Dinaciclib impairs recruitment of BRCA1 and RAD51 proteins to DSB sites and reduces homologous recombination repair of chromosome DSBs in MM cells}

As noted above, ABT-888 alone induces DSBs in MM-cell DNA, but these are efficiently repaired (19). Because CDKs 1, 2, and 5 (inhibited by dinaciclib) are known to play key roles in DSB repair, we asked whether dinaciclib might owe its synthetic lethality in combination with ABT-888, to blockage of HR repair allowing persistence of ABT-888induced DSBs. To this end, we used immunofluorescence detection and confocal microscopy to assess the levels of nuclear BRCA1 and RAD51 foci, which are indicators of HR repair, and of $\gamma \mathrm{H} 2 \mathrm{AX}$ foci (a marker of DSBs), after treatment of MM cells with DMSO control, dinaciclib, ABT-888, or dinaciclib plus ABT-888. As shown in Figure 2B, dinaciclib prevented repair of ABT-888-induced DNA damage, thus significantly reducing the fraction of cells with $\geq 5$ nuclear BRCA1 foci from $47 \%$ to $6 \%(p<0.0001)$ and $\geq 5$ nuclear RAD51 foci from $46 \%$ to $4 \%$ ( $p<0.0001)$, while causing a marked increase in the fraction of cells with $\geq 5$ nuclear $\gamma \mathrm{H} 2 \mathrm{AX}$ foci from $19 \%$ to $52 \%$ ( $p<0.0001)$. The integral of fluorescence signal per nucleus paralleled quite well the fraction of cells exceeding an arbitrary threshold (Figure 2). It is also noteworthy that dinaciclib treatment alone induced significant DNA damage, increasing the basal levels of cells with $\geq 5$ nuclear $\gamma \mathrm{H} 2 \mathrm{AX}$ foci from $20 \%$ to $35 \%$ ( $p<0.05$ ). This could be due to inhibition of CDK2, which is known to activate components of DSB-repair signaling, including formation of $\gamma \mathrm{H} 2 \mathrm{AX}$ foci (39-41).

We next assessed the direct impact of dinaciclib on the repair of chromosome DSBs via HR. The MM.1S cell line has robust HR-repair capability $(3,37)$ and so we used MM.1S cells bearing a chromosomally integrated DR-GFP reporter construct to measure HR repair as we described previously $(19,37)$. Typical results, shown in Figure 3, indicate $19 \% \mathrm{GFP}^{+}$cells (cells with HR-repair events following induction of a target-specific DSB) in control cells, but only $3 \%$ in dinaciclib-treated reporter cells - an $84 \%$ decrease in HR repair after dinaciclib treatment $(p<0.0001)$. Taken together, these findings demonstrate that dinaciclib impairs the recruitment of BRCA1 and RAD51 to DNA DSB sites, and subsequently inhibits HR repair of chromosomal DSBs.

\section{Dinaciclib lowers the mRNA levels of Rad51, its paralogs and Brca1, and reduces the protein levels of RAD51 and damage-induced phosphorylated BRCA1}

The RAD51 complex plays a central role in HR repair; RAD51 paralogs (RAD51B, RAD51C, RAD51D, XRCC2 and XRCC3) and BRCA1 promote RAD51 function during DSB repair (13). CDK inhibitors can induce p53-mediated downregulation of RAD51 at both the mRNA and protein levels, resulting in HR impairment and hypersensitivity of cells to DNA damaging agents $(42,43)$. CDK-mediated phosphorylation of BRCA1 on S1497 is required for the formation of BRCA1 and RAD51 repair foci following DNA damage (26, 27). We therefore assessed transcript levels of RAD51, its paralogs, and BRCA1 using RTqPCR; and we measured protein levels of RAD51 and pBRCA1 (S1497) on immunoblots before and after drug treatments. As shown in Figure 4A, dinaciclib alone ("SCH") significantly reduced mRNA levels of Rad51, Xrcc3, and Brcal, whereas ABT-888 alone had at best a marginal effect, only on Rad51d transcripts. The combination of dinaciclib and ABT-888, in contrast, strongly and very significantly suppressed transcripts of Rad51 (92\%), Rad51b (66\%), Rad51c (87\%) Xrcc2 (83\%), Xrcc3 (89\%), and Brcal (98\%) (each 
$p<0.0001)$. Only the effect on Rad51d was insignificant and relatively modest (57\%), apparently no different from that of ABT-888 alone (Figure 4A). Consequently, dinaciclib caused a significant and dose-dependent decrease in RAD51 protein levels (Figure 4, B-C). Doxorubicin, a topoisomerase II inhibitor that induces DNA DSBs, caused a 5-fold increase in pBRCA1 (S1497) levels relative to vehicle treatment. However, this effect was completely blocked by dinaciclib treatment (Figure 4, D-E). Taken together, we have demonstrated that dinaciclib treatment reduces the mRNA levels of Rad51, its paralogs, and Brcal as well as the protein levels of RAD51 and DNA damage-induced pBRCA1 (S1497). H929 cells responded to dinaciclib treatment quite similarly to MM.1S cells, with significant declines in transcripts for Rad51, its paralogs and Brcal (data not shown).

\section{Dinaciclib induces S-phase accumulation and reduces G2/M phase arrest}

Cell-cycle regulation is a major role of CDKs (44), which could in turn influence the expression of DNA-repair genes and the choice of DNA-repair pathways. Because we have demonstrated that dinaciclib disrupts HR repair, which occurs predominantly during the $S$ and $\mathrm{G} 2$ cell-cycle phases, we wanted to rule out possible preclusion of HR repair due to G1 arrest. We therefore used propidium iodide (PI) staining to assess the cell-cycle distribution after exposure to dinaciclib, and/or doxorubicin - which causes MM cell-cycle arrest in S and G2 (37). Treatment with doxorubicin (DOX) for $24 \mathrm{~h}$ caused cells to arrest in the S and G2 phases, as expected (Figure 5A), with very few cells in G1. Treatment with dinaciclib ( $\mathrm{SCH}$ ) alone caused moderate increases of cells in S phase (from $20 \%$ to $30 \%$ ), and $\mathrm{G} 1$ (from 50\% to 58\%), but a much more pronounced decrease (from $31 \%$ to $12 \%$ ) in G2/Mphase cells. This effect was observed for all of the MM cell lines tested (Suppl. Figure 2, AB). Since the total decrease of cells in the sum of S and G2 phases together was just 8-9\%, this would not account for the $>5$-fold impairment of HR by dinaciclib. The doxorubicininduced G2/M accumulation was completely reversed by dinaciclib, which restored $\mathrm{SCH}+$ DOX-treated cells to nearly the same distribution as SCH treatment alone, e.g., G2/M shifted from 63\% to 16\%; Figure 5). We also assessed the cell-cycle effects of ABT-888 with or without dinaciclib. Compared to control DMSO treatment, ABT-888 caused an increase in G2/M phase (from $31 \%$ to $45 \%$ ) and a corresponding decrease of cells in G1 phase (from 50\% to 33.6\%). The G2-phase accumulation was similarly reversed when dinaciclib was added to ABT-888, just as when it was combined with DOX (data not shown).

Because dinaciclib caused cells to accumulate in S phase, with a marked decrease in G2/M cells, but abrogated doxorubicin-induced G2/M-phase accumulation, we assessed the degree of S-phase arrest by BrdU incorporation, and stained cells for phospho-histone H3 (S10) to determine whether the $\mathrm{G} 2$ checkpoint was abrogated. In fact, very few cells treated with dinaciclib and/or doxorubicin underwent DNA synthesis (i.e., the BrdU-positive fraction of cells in S phase was <2\%; Figure 5, D and F). Thus the S phase checkpoint was apparently not inactivated by dinaciclib. Phosphorylated histone $\mathrm{H} 3$ (S10) (pHH3) is a marker of mitotic cells, and often used to distinguish cells in G2 and M phases and thus to determine G2 checkpoint abrogation. Cells treated with dinaciclib and/or doxorubicin showed very little pHH3 staining $(<0.015 \%)$, indicating that cells in the G2/M phase were almost entirely arrested in G2. Thus there was little G2 checkpoint abrogation. 
We thus conclude from our cell-cycle data that the impairment of HR by dinaciclib could not be explained by G1 arrest. On the contrary, accumulation of cells in S phase could enhance drug cytotoxicity since cells are most vulnerable to killing by cytotoxic drugs such as anthracyclines (e.g. doxorubicin) during the $\mathrm{S}$ phase (45).

\section{Co-treatment with dinaciclib and ABT-888 delays tumor growth and significantly prolongs survival of SCID mice bearing MM xenografts}

We next evaluated the effects of ABT-888 $(50 \mathrm{mg} / \mathrm{kg}$ by oral gavage twice daily, 5 days per week) and/or dinaciclib (35 mg/kg i.p, twice per week) on the growth of MM.1S cells as subcutaneous (SC) tumors, after SC inoculation in male SCID mice. On the third week of treatment, tumor volume had increased 20 -fold in mice exposed only to vehicle $(6 \%$ DMSO), and 19-fold for ABT-888, but 9-fold with dinaciclib ( $p<0.01$ compared with untreated controls or ABT-888 treatment), and just 1.7-fold for co-treatment with dinaciclib and ABT-888 ( $p \unlhd 0.012$, compared to either single agent; Figure 6). The in vivo effects were thus in line with the in vitro data. Log-rank tests for Kaplan-Meier survival curves also showed a significant increase in pre-morbid survival ( $p \unlhd 0.004$; Figure 6 ) for the dinaciclib plus ABT-888 co-treated group relative to either single agent. Although we observed mild diarrhea and slight weight loss in mice treated with dinaciclib, they were overall very healthy and active and did not show signs of discomfort. To further assess whether the downregulation of DNA repair genes after dinaciclib treatment also occurs in vivo, when tumors had reached the pre-determined size limit $\left(2000 \mathrm{~mm}^{3}\right)$, we repeated the drug treatments for $24 \mathrm{~h}$, then excised the tumors and preserved them in RNAlater ${ }^{\mathrm{TM}}$ for RT-qPCR analyses on total RNA. We observed significant downregulation of mRNA levels for Rad51, $X r c c 3$ and Brcal in groups treated with dinaciclib alone or combined with ABT-888 (Supplementary Figure 3). The reductions in transcript levels for Rad51b, Rad51c, Rad51d, and $X r c c 2$ were not statistically significant after dinaciclib, and ABT-888 treatment had no significant impact on the expression of any of the genes tested.

In summary, our in vivo data on tumor growth and transcript levels were entirely consistent the in vitro data (although somewhat more variable), lending further support to our hypothesis that contextual synthetic lethality to MM cells can be achieved with the combination of dinaciclib and ABT-888.

\section{Discussion}

Unlike normal bone marrow plasma cells and normal B cells, MM cell lines and patient plasma cells display marked chromosomal instability (2) and pervasive DNA damage (21). These implicate aberrant DNA repair pathways in the disease. We have previously reported that HR frequency is elevated in MM and contributes to genomic instability and disease progression (3), while other studies have shown that PARP1 is overexpressed and correlates with shortened survival (19). Elevated HR and PARP1 activities may compensate for certain defects in components of the DNA damage-response machinery, such as NHEJ (4) and MMR $(5,46)$; thus myeloma cells may be especially dependent on PARP1 and HR for survival $(19,46)$. Recent studies have identified critical roles for CDKs in HR repair, and specific depletion of CDK1 or CDK5 has been shown to cause hypersensitivity of tumor 
cells to PARP inhibitors (26-28). We have demonstrated in this study that the CDK inhibitor dinaciclib disrupts HR function and causes a contextual synthetic lethality of MM cells when combined with the PARP1/2 inhibitor ABT-888, while sparing normal peripheral blood CD $19^{+}$B cells.

We demonstrated that the survival of MM cell lines is not significantly impaired by treatment with the PARP inhibitor ABT-888 alone. This is not surprising, considering the elevated state of HR in MM cells (3). Moreover, previous studies demonstrated that ABT-888 does not affect MM-cell survival because their HR efficiently repairs the induced DSBs (19). Although we found that dinaciclib alone significantly decreased MM cell viability, a synergistic lethal effect ensues when it is combined with the PARP inhibitor ABT-888, leading to a significantly greater loss of viability relative to these agents given singly, and even relative to the product of their individual effects. This synthetic-lethal effect was recapitulated in MM xenografts in SCID mice, where tumor growth was significantly delayed and survival prolonged for the combination compared to individual agents. Intriguingly, a synthetic-lethal effect was not observed in normal B cells. As mentioned above, MM cells have ongoing DNA damage and defects in DNA-repair pathways, leading to especially high dependence on HR and PARP activities. In contrast, normal cells have intact DNA-repair pathways and accumulate little or no unrepaired DNA damage. Thus, inhibiting PARP and HR would be expected to show preferential toxicity for MM cells. This provides a good rationale for combining dinaciclib and ABT- 888 in MM treatment, since this would widen the therapeutic window. With regard to the differential sensitivity to dinaciclib, of MM cell lines relative to normal B cells, deregulation of the cell cycle and the high proliferation of MM cell lines could increase their sensitivity to CDK inhibitors compared to normal cells. In addition to PARP inhibition, we found that dinaciclib produced synergistic effects in combination with doxorubicin, a topoisomerase II inhibitor. Previous studies have shown that dinaciclib enhances the antitumor effects of gemcitabine, an agent known to cause replication-fork collapse and DSB formation, which requires HR repair (47). Also, Raje et al., reported that seliciclib (a CDK inhibitor with targets similar to dinaciclib's), produced synergistic toxicity for MM cells when combined with doxorubicin (48). These studies are consistent with our observations that dinaciclib potentiates the antiMM effects of ABT-888, which causes replication-fork collapse and subsequent DSB formation, and doxorubicin, which induces DSBs.

We posited that HR deficiency and the consequent increase in DNA damage are the basis for dinaciclib-induced sensitivity of MM cells to PARP inhibition. In keeping with this, we demonstrated that ABT-888 treatment induced BRCA1 and RAD51 foci, which are indicative of proficient HR repair, thereby resulting in very little DNA damage or few $\gamma \mathrm{H} 2 \mathrm{AX}$ foci. On the contrary, upon combining ABT- 888 with dinaciclib, the reverse was true: fewer BRCA1 and RAD51 foci and increased $\gamma \mathrm{H} 2 \mathrm{AX}$ foci were observed. The inhibition of HR repair was further supported by our GFP-based HR assays, which showed that dinaciclib directly blocks HR repair of chromosomal DSBs. To pursue this we examined the basis for reduced BRCA1 and RAD51 foci induced by dinaciclib, and found that dinaciclib impairs phosphorylation of BRCA1 on ser1497, the expected CDK phosphorylation site $(25,26)$. This modification is required for BRCA1 focus formation and RAD51-mediated HR repair, whereas inhibition of this phosphorylation sensitizes cancer 
cells to PARP inhibitors $(26,33)$. Dinaciclib also decreased both Rad51 mRNA and protein levels of RAD51, a core protein essential for HR repair whose depletion sensitizes tumor cells to PARP inhibition (49). Additionally, the transcript levels of Rad51 paralogs as well as Brcal were reduced by dinaciclib. These genes encode proteins that promote RAD51mediated HR repair. The decrease in RAD51 transcripts and protein was in line with previous findings that CDK inhibitors mediate p53-dependent downregulation of RAD51 at both the transcript and protein levels, and sensitize cancer cells to DNA damaging agents $(42,43)$. We therefore conclude that these effects of dinaciclib led to the impaired HR function and the subsequent sensitivity of MM cells to PARP inhibition as well as doxorubicin. Moreover, we have previously reported that disruption of RAD51 expression, via siRNA or a small-molecule inhibitor, impairs HR function and sensitizes MM cells (37).

We also note that CDK1/2-mediated phosphorylation of CtIP is required for HR repair, and inhibition of these CDKs via triapine has been demonstrated to compromise HR repair and sensitize ovarian cancer cells to inhibitors of PARP and topoisomerases (50). It is therefore possible that dinaciclib also acts through CtIP to reduce HR repair in addition to the pathways we investigated. Finally, we have demonstrated that the HR-repair inhibitory effects of dinaciclib are not cell-cycle-related, since dinaciclib did not induce cell-cycle phase arrest in G1, which if it occurred could preclude HR repair. It remains to be determined which specific CDK(s) inhibited by dinaciclib account for its HR-inhibitory effects in MM cells.

To summarize, we have used a CDK inhibitor, dinaciclib, to disrupt BRCA1 and RAD51 functions, which impaired the ability of MM cells to repair chromosome DSBs via HR. This approach selectively sensitizes MM cells to the PARP1/2 inhibitor ABT-888, in a manner that (based on similar synthetic lethality for other drug combinations) appears to be mediated by dinaciclib's disruption of HR repair. Since CDKs, PARP1 and HR are deregulated in MM and have been designated as therapeutic targets, our findings provide a strong rationale for combining dinaciclib with PARP inhibitors in targeting MM cells, and a basis for further investigations of their safety and efficacy in MM patients.

\section{Supplementary Material}

Refer to Web version on PubMed Central for supplementary material.

\section{Acknowledgments}

Financial Support: This study was supported by a VA Merit grant (PI: R.J. Shmookler Reis), and NIH grants P01 CA055819-20 (PI: B. Barlogie [PL: S. Yaccoby]) and P30 AG028718 (PI: J. Wei [CL: R.J. Shmookler Reis]).

\section{References}

1. Morgan GJ, Walker BA, Davies FE. The genetic architecture of multiple myeloma. Nat Rev Cancer. 2012; 12:335-48. [PubMed: 22495321]

2. Bergsagel PL, Kuehl WM. Chromosome translocations in multiple myeloma. Oncogene. 2001; 20:5611-22. [PubMed: 11607813] 
3. Shammas MA, Shmookler Reis RJ, Koley H, Batchu RB, Li C, Munshi NC. Dysfunctional homologous recombination mediates genomic instability and progression in myeloma. Blood. 2009; 113:2290-7. [PubMed: 19050310]

4. Yang C, Betti C, Singh S, Toor A, Vaughan A. Impaired NHEJ function in multiple myeloma. Mutation research. 2009; 660:66-73. [PubMed: 19028508]

5. Velangi MR, Matheson EC, Morgan GJ, Jackson GH, Taylor PR, Hall AG, et al. DNA mismatch repair pathway defects in the pathogenesis and evolution of myeloma. Carcinogenesis. 2004; 25:1795-803. [PubMed: 15142887]

6. Lieber MR. The mechanism of human nonhomologous DNA end joining. The Journal of biological chemistry. 2008; 283:1-5. [PubMed: 17999957]

7. Rothkamm K, Kruger I, Thompson LH, Lobrich M. Pathways of DNA double-strand break repair during the mammalian cell cycle. Molecular and cellular biology. 2003; 23:5706-15. [PubMed: 12897142]

8. Lee JH, Paull TT. ATM activation by DNA double-strand breaks through the Mre11-Rad50-Nbs1 complex. Science. 2005; 308:551-4. [PubMed: 15790808]

9. Lou Z, Minter-Dykhouse K, Franco S, Gostissa M, Rivera MA, Celeste A, et al. MDC1 maintains genomic stability by participating in the amplification of ATM-dependent DNA damage signals. Molecular cell. 2006; 21:187-200. [PubMed: 16427009]

10. Cortez, D.; Wang, Y.; Qin, J.; Elledge, SJ. Science. Vol. 286. New York, NY: 1999. Requirement of ATM-dependent phosphorylation of brca1 in the DNA damage response to double-strand breaks; p. 1162-6.

11. Yun MH, Hiom K. CtIP-BRCA1 modulates the choice of DNA double-strand-break repair pathway throughout the cell cycle. Nature. 2009; 459:460-3. [PubMed: 19357644]

12. Yu X, Chen J. DNA damage-induced cell cycle checkpoint control requires CtIP, a phosphorylation-dependent binding partner of BRCA1 C-terminal domains. Molecular and cellular biology. 2004; 24:9478-86. [PubMed: 15485915]

13. Sung P, Klein H. Mechanism of homologous recombination: mediators and helicases take on regulatory functions. Nature reviews Molecular cell biology. 2006; 7:739-50. [PubMed: 16926856]

14. Helleday T. The underlying mechanism for the PARP and BRCA synthetic lethality: clearing up the misunderstandings. Molecular oncology. 2011; 5:387-93. [PubMed: 21821475]

15. Farmer H, McCabe N, Lord CJ, Tutt AN, Johnson DA, Richardson TB, et al. Targeting the DNA repair defect in BRCA mutant cells as a therapeutic strategy. Nature. 2005; 434:917-21. [PubMed: 15829967]

16. Arnaudeau C, Lundin C, Helleday T. DNA double-strand breaks associated with replication forks are predominantly repaired by homologous recombination involving an exchange mechanism in mammalian cells. J Mol Biol. 2001; 307:1235-45. [PubMed: 11292338]

17. Patel AG, Sarkaria JN, Kaufmann SH. Nonhomologous end joining drives poly(ADP-ribose) polymerase (PARP) inhibitor lethality in homologous recombination-deficient cells. Proceedings of the National Academy of Sciences of the United States of America. 2011; 108:3406-11. [PubMed: 21300883]

18. Bryant HE, Schultz N, Thomas HD, Parker KM, Flower D, Lopez E, et al. Specific killing of BRCA2-deficient tumours with inhibitors of poly(ADP-ribose) polymerase. Nature. 2005; 434:913-7. [PubMed: 15829966]

19. Neri P, Ren L, Gratton K, Stebner E, Johnson J, Klimowicz A, et al. Bortezomib-induced "BRCAness" sensitizes multiple myeloma cells to PARP inhibitors. Blood. 2011; 118:6368-79. [PubMed: 21917757]

20. Tai YT, Teoh G, Lin B, Davies FE, Chauhan D, Treon SP, et al. Ku86 variant expression and function in multiple myeloma cells is associated with increased sensitivity to DNA damage. J Immunol. 2000; 165:6347-55. [PubMed: 11086072]

21. Walters DK, Wu X, Tschumper RC, Arendt BK, Huddleston PM, Henderson KJ, et al. Evidence for ongoing DNA damage in multiple myeloma cells as revealed by constitutive phosphorylation of H2AX. Leukemia. 2011; 25:1344-53. [PubMed: 21566653] 
22. Bergsagel PL, Kuehl WM, Zhan F, Sawyer J, Barlogie B, Shaughnessy J Jr. Cyclin D dysregulation: an early and unifying pathogenic event in multiple myeloma. Blood. 2005; 106:296-303. [PubMed: 15755896]

23. Canavese M, Santo L, Raje N. Cyclin dependent kinases in cancer: potential for therapeutic intervention. Cancer biology \& therapy. 2012; 13:451-7. [PubMed: 22361734]

24. Kumar SK, LaPlant B, Chng WJ, Zonder J, Callander N, Fonseca R, et al. Dinaciclib, a novel CDK inhibitor, demonstrates encouraging single agent activity in patients with relapsed multiple myeloma. Blood. 2014; 13:2014-05.

25. Ruffner H, Jiang W, Craig AG, Hunter T, Verma IM. BRCA1 is phosphorylated at serine 1497 in vivo at a cyclin-dependent kinase 2 phosphorylation site. Molecular and cellular biology. 1999; 19:4843-54. [PubMed: 10373534]

26. Johnson N, Cai D, Kennedy RD, Pathania S, Arora M, Li YC, et al. Cdk1 participates in BRCA1dependent $S$ phase checkpoint control in response to DNA damage. Molecular cell. 2009; 35:32739. [PubMed: 19683496]

27. Johnson N, Li YC, Walton ZE, Cheng KA, Li D, Rodig SJ, et al. Compromised CDK1 activity sensitizes BRCA-proficient cancers to PARP inhibition. Nature medicine. 2011; 17:875-82.

28. Turner NC, Lord CJ, Iorns E, Brough R, Swift S, Elliott R, et al. A synthetic lethal siRNA screen identifying genes mediating sensitivity to a PARP inhibitor. The EMBO journal. 2008; 27:136877. [PubMed: 18388863]

29. Zhu YX, Tiedemann R, Shi CX, Yin H, Schmidt JE, Bruins LA, et al. RNAi screen of the druggable genome identifies modulators of proteasome inhibitor sensitivity in myeloma including CDK5. Blood. 2011; 117:3847-57. [PubMed: 21289309]

30. Tian B, Yang Q, Mao Z. Phosphorylation of ATM by Cdk5 mediates DNA damage signalling and regulates neuronal death. Nature cell biology. 2009; 11:211-8. [PubMed: 19151707]

31. Biswas AK, Johnson DG. Transcriptional and Nontranscriptional Functions of E2F1 in Response to DNA Damage. Cancer Res. 2012; 72:13-7. [PubMed: 22180494]

32. Martinez-Cardus A, Martinez-Balibrea E, Bandres E, Malumbres R, Gines A, Manzano JL, et al. Pharmacogenomic approach for the identification of novel determinants of acquired resistance to oxaliplatin in colorectal cancer. Mol Cancer Ther. 2009; 8:194-202. [PubMed: 19139129]

33. Kerr AR, Warnakulasuriya S, Mighell AJ, Dietrich T, Nasser M, Rimal J, et al. A systematic review of medical interventions for oral submucous fibrosis and future research opportunities. Oral diseases. 2011; 17(Suppl 1):42-57. [PubMed: 21382138]

34. Jorda R, Havlicek L, McNae IW, Walkinshaw MD, Voller J, Sturc A, et al. Pyrazolo[4,3d]pyrimidine bioisostere of roscovitine: evaluation of a novel selective inhibitor of cyclindependent kinases with antiproliferative activity. Journal of medicinal chemistry. 2011; 54:2980 93. [PubMed: 21417417]

35. Raghavan P, Tumati V, Yu L, Chan N, Tomimatsu N, Burma S, et al. AZD5438, an inhibitor of Cdk1, 2, and 9, enhances the radiosensitivity of non-small cell lung carcinoma cells. International journal of radiation oncology, biology, physics. 2012; 84:e507-14.

36. Parry D, Guzi T, Shanahan F, Davis N, Prabhavalkar D, Wiswell D, et al. Dinaciclib (SCH 727965), a novel and potent cyclin-dependent kinase inhibitor. Molecular cancer therapeutics. 2010; 9:2344-53. [PubMed: 20663931]

37. Alagpulinsa DA, Ayyadevara S, Shmookler Reis RJ. A Small-Molecule Inhibitor of RAD51 Reduces Homologous Recombination and Sensitizes Multiple Myeloma Cells to Doxorubicin. Front Oncol. 2014; 4

38. LeBlanc R, Catley LP, Hideshima T, Lentzsch S, Mitsiades CS, Mitsiades N, et al. Proteasome inhibitor PS-341 inhibits human myeloma cell growth in vivo and prolongs survival in a murine model. Cancer Res. 2002; 62:4996-5000. [PubMed: 12208752]

39. Zhu Y, Alvarez C, Doll R, Kurata H, Schebye XM, Parry D, et al. Intra-S-phase checkpoint activation by direct CDK2 inhibition. Molecular and cellular biology. 2004; 24:6268-77. [PubMed: 15226429]

40. Sherr CJ, Roberts JM. CDK inhibitors: positive and negative regulators of G1-phase progression. Genes Dev. 1999; 13:1501-12. [PubMed: 10385618] 
41. Deans AJ, Khanna KK, McNees CJ, Mercurio C, Heierhorst J, McArthur GA. Cyclin-dependent kinase 2 functions in normal DNA repair and is a therapeutic target in BRCA1-deficient cancers. Cancer Res. 2006; 66:8219-26. [PubMed: 16912201]

42. Kotala V, Uldrijan S, Horky M, Trbusek M, Strnad M, Vojtesek B. Potent induction of wild-type p53-dependent transcription in tumour cells by a synthetic inhibitor of cyclin-dependent kinases. Cell Mol Life Sci. 2001; 58:1333-9. [PubMed: 11577989]

43. Ambrosini G, Seelman SL, Qin LX, Schwartz GK. The cyclin-dependent kinase inhibitor flavopiridol potentiates the effects of topoisomerase I poisons by suppressing Rad51 expression in a p53-dependent manner. Cancer Res. 2008; 68:2312-20. [PubMed: 18381438]

44. Norbury C, Nurse P. Animal cell cycles and their control. Annual review of biochemistry. 1992; 61:441-70.

45. Hill BT, Whelan RD. A comparison of the lethal and kinetic effects of doxorubicin and $4^{\prime}$-epidoxorubicin in vitro. Tumori. 1982; 68:29-37. [PubMed: 6951322]

46. Elliott B, Jasin M. Repair of double-strand breaks by homologous recombination in mismatch repair-defective mammalian cells. Molecular and cellular biology. 2001; 21:2671-82. [PubMed: 11283247]

47. Feldmann G, Mishra A, Bisht S, Karikari C, Garrido-Laguna I, Rasheed Z, et al. Cyclin-dependent kinase inhibitor Dinaciclib (SCH727965) inhibits pancreatic cancer growth and progression in murine xenograft models. Cancer Biol Ther. 2011; 12:598-609. [PubMed: 21768779]

48. Raje N, Kumar S, Hideshima T, Roccaro A, Ishitsuka K, Yasui H, et al. Seliciclib (CYC202 or Rroscovitine), a small-molecule cyclin-dependent kinase inhibitor, mediates activity via downregulation of Mcl-1 in multiple myeloma. Blood. 2005; 106:1042-7. [PubMed: 15827128]

49. McCabe N, Turner NC, Lord CJ, Kluzek K, Bialkowska A, Swift S, et al. Deficiency in the repair of DNA damage by homologous recombination and sensitivity to poly(ADP-ribose) polymerase inhibition. Cancer Res. 2006; 66:8109-15. [PubMed: 16912188]

50. Lin ZP, Ratner ES, Whicker ME, Lee Y, Sartorelli AC. Triapine disrupts CtIP-mediated homologous recombination repair and sensitizes ovarian cancer cells to PARP and topoisomerase inhibitors. Mol Cancer Res. 2014; 12:381-93. [PubMed: 24413181] 

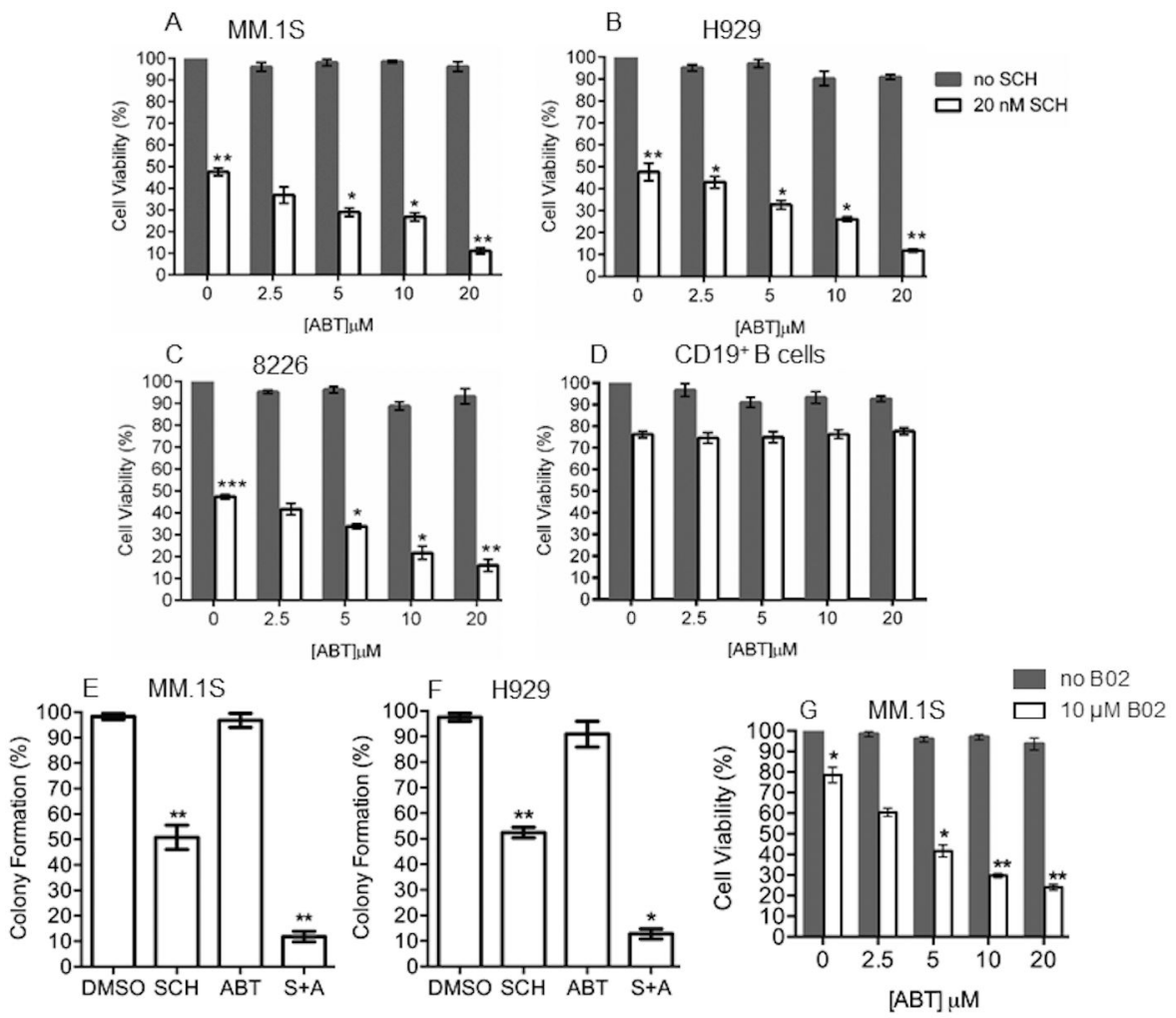

Figure 1. Synthetic lethality of MM cells, but not normal B cells, co-treated with the CDK inhibitor dinaciclib and the PARP1/2 inhibitor ABT-888

MM cell lines (A-C) or CD19 ${ }^{+} \mathrm{B}$ cells from normal peripheral blood (D) were treated with dinaciclib (SCH), ABT-888 (ABT), or the two combined $(\mathrm{S}+\mathrm{A})$, or B02 \pm ABT $(\mathrm{G})$, at the indicated doses. Cell viability was measured by WST-1 assay after 72 hours. Clonogenic survival assays were also run for MM.1S (E) and H929 (F) myeloma cells treated with 20$\mathrm{nM}$ SCH, 20- $\mu \mathrm{M}$ ABT, or their combination. Mean survival or colony formation $\pm \mathrm{SEM}$ are shown for 3 replicate experiments normalized to the untreated controls. *, ** and *** indicate $p<0.05,0.01$ and 0.001 , respectively, for 2-tailed paired $t$-tests against SCH treatment alone (open bar, DOX $=0 \mathrm{nM}$ ), with the exception of SCH treatment, which was compared to its vehicle-only control (i.e. contrasting the filled vs. open bar at DOX $=0 \mathrm{nM}$ ). 


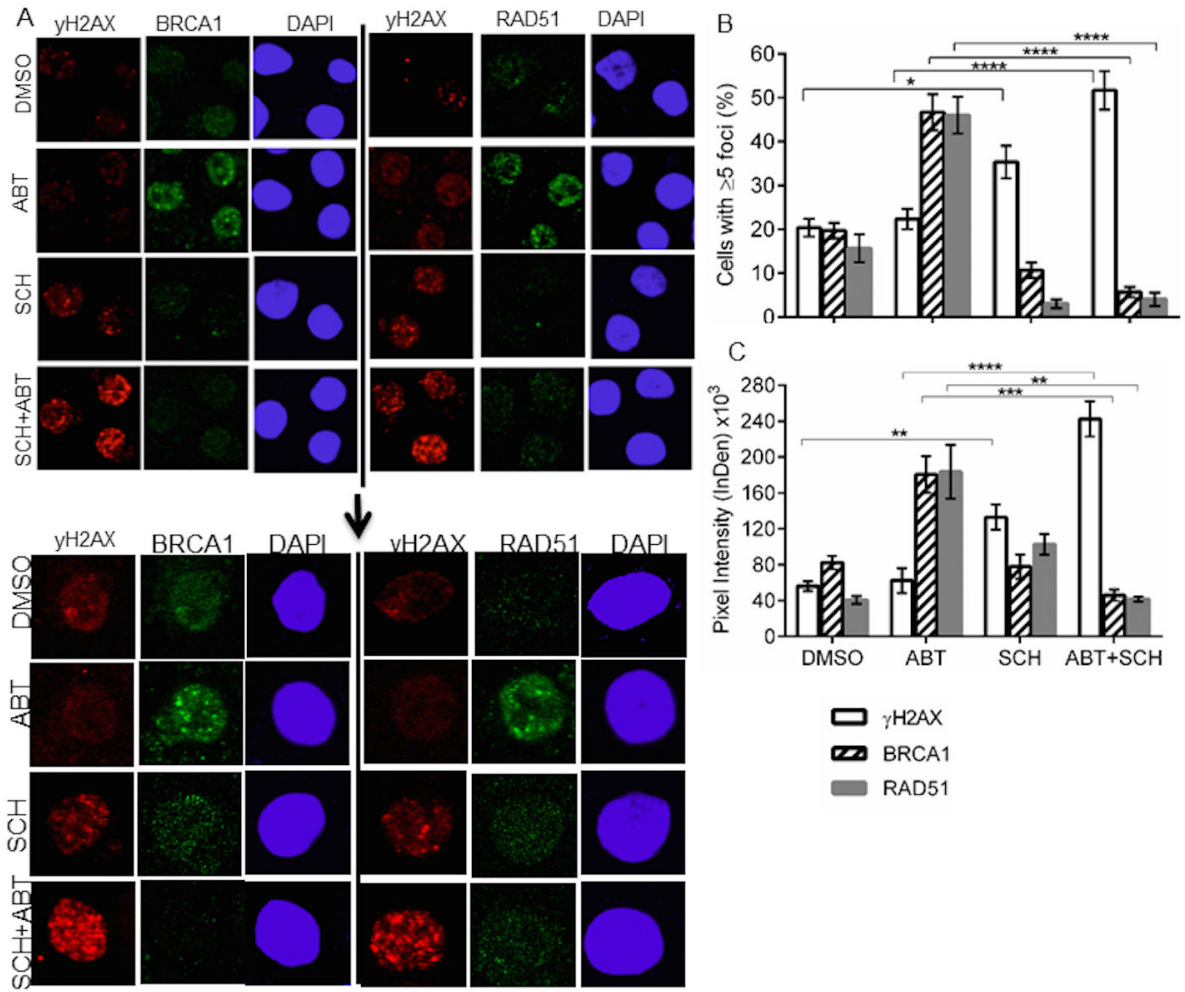

Figure 2. Dinaciclib impairs recruitment of BRCA1 and RAD51 proteins to DSB sites MM.1S cells, exposed $24 \mathrm{~h}$ to DMSO, dinaciclib (SCH; $20 \mathrm{nM}$ ), ABT-888 (ABT; $20 \mu \mathrm{M})$ or $\mathrm{SCH}+\mathrm{ABT}$, were examined by immunofluorescence to identify foci, and DAPI staining to define nuclei. (A) Representative images of RAD51 and $\gamma \mathrm{H} 2 \mathrm{AX}$ foci in cells exposed to drugs indicated at left. (B) Mean percent of cells with $\geq 5$ RAD51 or $\gamma \mathrm{H} 2 \mathrm{AX}$ foci, \pm SEM, after the exposures indicated; data were combined from three experiments. (C) Integrated signal intensity per nucleus, after subtraction of peripheral background. $(\mathrm{B}, \mathrm{C}) *$ ***,*** and $* * * *$ indicate $p<0.05, p<0.01, p<0.001$ and $p<0.0001$, respectively, for 2-tailed $t$-tests on the effect of drug treatment relative to DMSO (vehicle). 

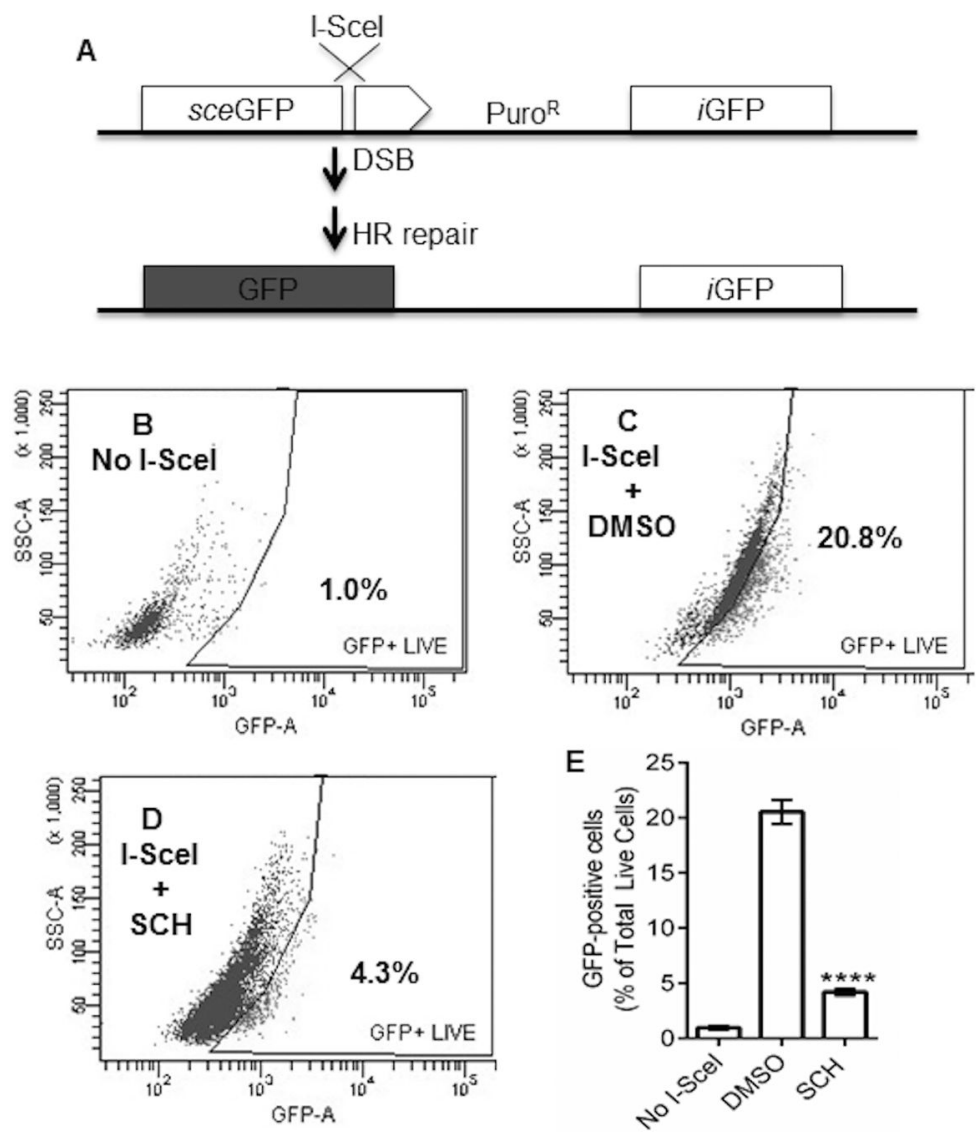

Figure 3. Dinaciclib reduces HR repair of chromosome DSBs in MM cells (A) HR repair reporter substrate: The HR reporter DR-GFP is stably integrated in the genome of the MM.1S myeloma cell line (MM.1S-DR-GFP). The sceGFP is a GFP gene that contains an I-SceI endonuclease site within the coding region; its cleavage in vivo and repair by $\mathrm{HR}$, using the downstream $i G F P$ repeat as template, results in $\mathrm{GFP}^{+}$cells. (B-D) Examples of flow-cytometric analysis of MM.1S-DR-GFP cells, wherein GFP fluorescence ( $x$-axis signal) beyond the control boundary (segmented line) indicates HR repair. (E) Summary of combined data from runs such as those illustrated in (B-D), for cells without ISceI transfection (mock-treated), cells treated with vehicle (DMSO), or 20-nM SCH, for $24 \mathrm{~h}$ after transient transfection with I-SceI expression adenovirus (AdNUGS24i). HR data combined from three experiments are presented as mean \pm SEM. Statistical significance of the difference between groups ( $\mathrm{SCH} v s$. DMSO, each $n=3$ ) was determined by a two-tailed $t$ -test; $* * * * p<0.0001$. 
A

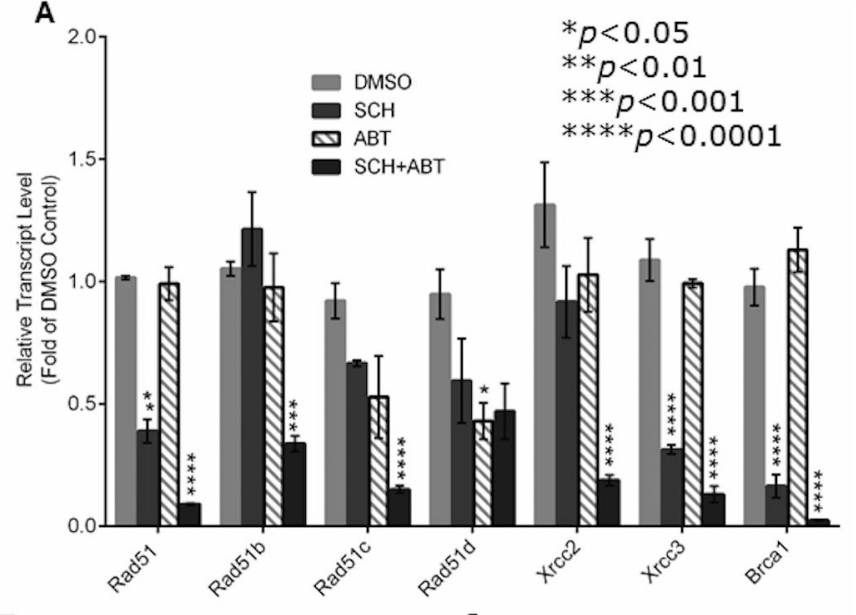

B

\section{C}

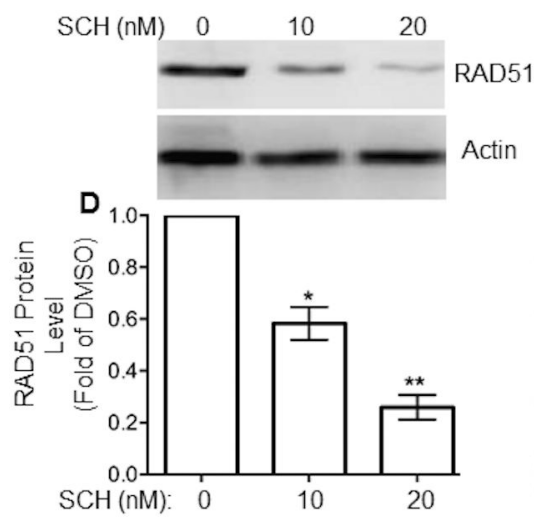

$\mathrm{SCH}$
$\mathrm{DOX}$ pBRCA1(\$1497)
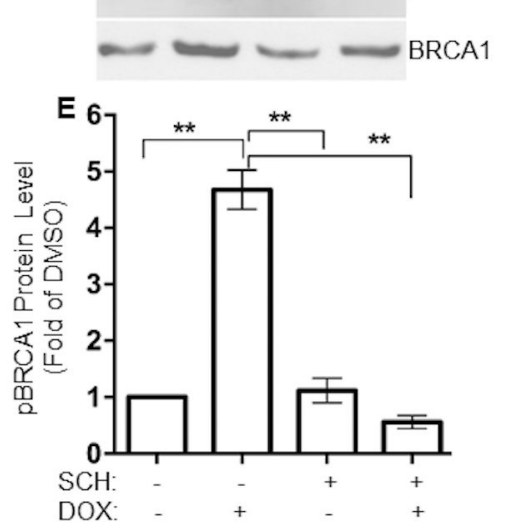

Figure 4. Dinaciclib reduces the mRNA levels of RAD51, its paralogs and BRCA1, and reduces the protein levels of RAD51 and phosphorylated BRCA1 (ser1497)

(A) Reduction in the quantity of mRNA for Rad51, its paralogs and Brcal as determined by RT-qPCR; (B, C) dose-dependent decrease in RAD51 protein levels; and (D, E) blockage of doxorubicin-induced phosphorylation of BRCA1 on S1497, as shown in western blots. Data were combined from at least two independent experiments (with $n=3$ per group in each experiment), shown as means \pm SEM. $*, * *, * * *$ and $* * * *$ indicate $p<0.05,0.01,0.001$ and 0.0001 , respectively, by 2-tailed $t$ tests for the significance of differences between each treatment and its control (DMSO). 
A
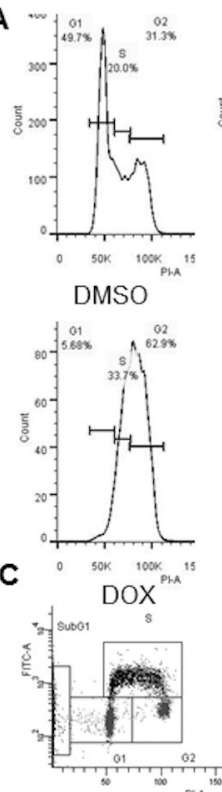

DOX

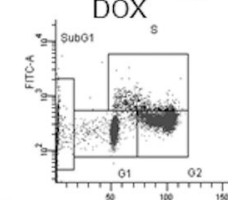

E

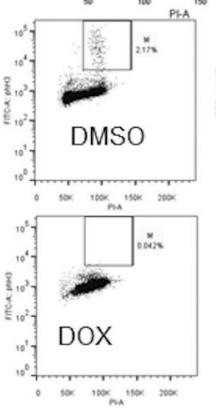

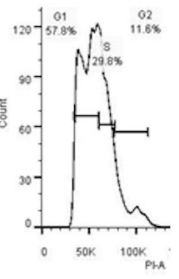
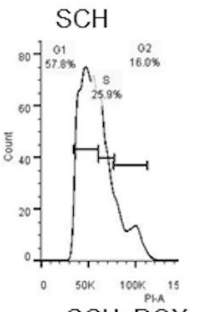

SCH+DOX
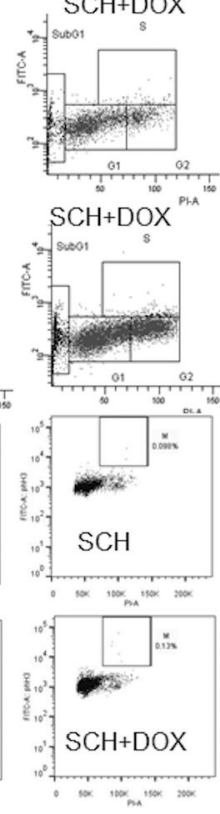

B

$\mathrm{G} 1 \square \mathrm{S} \square \mathrm{G} 2 \mathrm{M}$

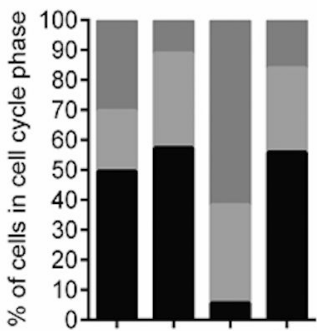

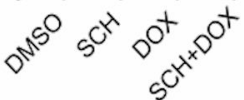
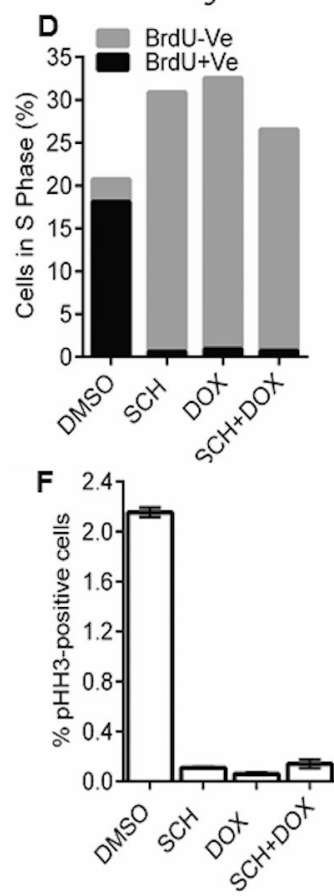

Figure 5. Dinaciclib induces S-phase accumulation and reduces G2/M-phase accumulation MM.1S cells were treated as indicated for $24 \mathrm{~h}$, and $(\mathbf{A}, \mathbf{B})$ cell-cycle distribution was determined by propidium iodide (PI) staining. $($ C, D) BrdU incorporation was assessed to determine the percent of cells with S phase DNA content (as determined by PI staining) that are BrdU-positive or -negative. (E, F) After the indicated treatments, cells were co-stained for DNA content with PI, and for phospho-histone H3 (S10) with Alexa Fluor 488conjugated antibody to phospho-histone H3 (S10). Panels (E) show representative flowcytometry scatter plots. (F) Averaged results are shown from independent experiments. Each treatment differed from the DMSO control by 2-tailed $t$ test at least $p<0.001$. 

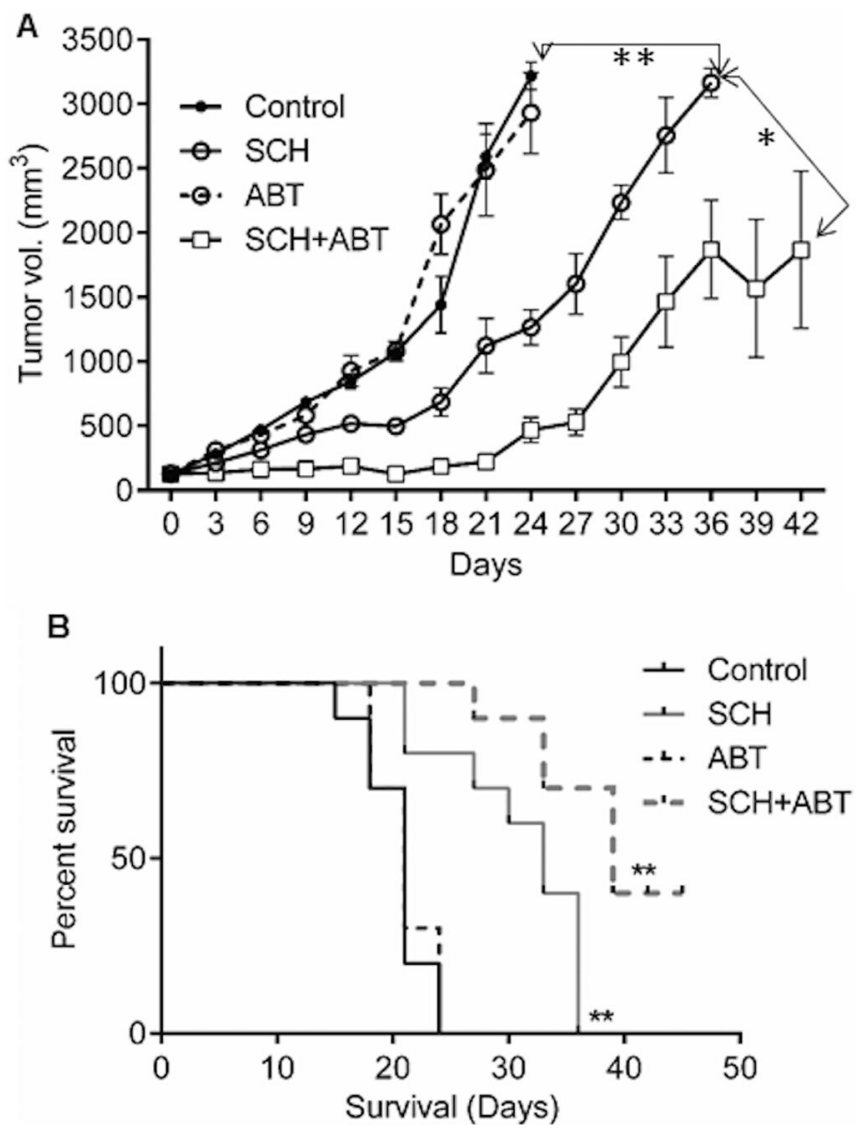

Figure 6. Effect of dinaciclib and ABT-888 on tumor growth and survival in SCID mice bearing MM xenografts

CB.17 SCID mice were inoculated subcutaneously with $5 \times 10^{6} \mathrm{MM}$.1S cells, and mice were randomized into 4 groups $(\mathrm{n}=10)$ when tumors became measurable $\left(100-150 \mathrm{~mm}^{3}\right)$. Groups were treated with vehicle (DMSO), dinaciclib ( $\mathrm{SCH} ; 35 \mathrm{mg} / \mathrm{kg}$ i.p twice weekly), ABT-888

(ABT; $50 \mathrm{mg} / \mathrm{kg}$ p.o. twice daily, five times a week) or the combined treatments of SCH and ABT-888. Tumor volumes measured every 3 days as indicated in Materials and Methods. (A) Tumor volumes were evaluated on the third week of treatment and significance of differences in tumor volume among groups was determined by ANOVA followed by the Tukey's post hoc test; * and ** indicate $p<0.05$ and 0.01 , respectively, for the indicated comparisons. (B) Kaplan-Meier survival was evaluated from the day treatment started to the day tumor volume reached the pre-determined termination point (see Materials and Methods). Significance of difference in survival between groups was determined by logrank test; * and $* *$ indicate $p<0.05$ and 0.01 , respectively, relative to DMSO control. 